\title{
Influence d'une construction alternative sur l'interprétation d'un pronom chez les enfants francophones au développement typique et atypique du langage
}

\author{
Saveria Colonna*1, Coralie Vincent ${ }^{1}$, Lisa Pinvidic $^{2}$, et Sarah Schimke $^{3}$ \\ ${ }^{1}$ University Paris 8/CNRS, France \\ ${ }^{2}$ Département d'orthophonie (DUEFO) Pitié-Salpêtrière, Université Pierre et Marie Curie \\ ${ }^{3}$ Université de Dortmund
}

\begin{abstract}
Résumé. Dans une expérience d'enregistrement des mouvements oculaires pendant l'exploration de référents présentés visuellement, nous avons observé les stratégies d'interprétation d'un pronom anaphorique chez des enfants francophones de 4 ans au développement langagier typique et des enfants avec retard de langage. Nous avons comparé leur interprétation d'une construction avec un pronom ambigu telle que "Le lapin chatouille le renard avant qu'il imagine..." avec leur interprétation de la construction alternative non ambiguë "Le lapin chatouille le renard avant d'imaginer...". Les résultats montrent qu'alors que les enfants au développement typique interprètent différemment les 2 constructions, ce n'est pas le cas des enfants avec retard de langage. En revanche, même s'ils commencent à distinguer les 2 constructions, les enfants sans retard ne les interprètent pas encore tout à fait comme les adultes. Pour rendre compte de ces résultats, nous proposons que, contrairement aux adultes, les enfants de 4 ans n'ont pas encore été suffisamment exposés à la construction infinitive non ambiguë pour qu'elle influence leur interprétation de la construction avec un pronom ambigu.
\end{abstract}

\begin{abstract}
Influence of an alternative construction on pronoun interpretation in French-speaking children with typical and atypical language development. In a visual world eye-tracking experiment, we observed strategies for interpreting an anaphoric pronoun in 4-year-old French-speaking children with typical and atypical language development. We compared their interpretation of a construction with an ambiguous pronoun such as "The rabbit tickles the fox before he imagines..." with their interpretation of the unambiguous alternative construction "The rabbit tickles the fox before imagining...". The results show that while typically developing children interpret both constructions differently, this is not the case for children with language delay. On the other hand, even if they begin to distinguish the two constructions, children without language delay do not yet interpret them in an adult-like way. To account for these results, we propose that, unlike adults, 4-year-olds have not yet been sufficiently exposed to the unambiguous infinite construction to influence their interpretation of the construction with an ambiguous pronoun.
\end{abstract}

\section{Introduction}

Pour interpréter un pronom, nous savons que les adultes prennent rapidement en compte différents indices linguistiques dont (entre autres) la récence linéaire, la structure syntaxique des énoncés, le sémantisme de certains verbes et le statut informationnel des référents potentiels (pour une revue, voir par exemple, de la Fuente, Hemforth, Colonna \& Schimke, 2016). Or, le poids de ces indices varie suivant l'âge, la trajectoire développementale et la langue des locuteurs. Prédire la pondération de ces différents indices suivant les propriétés des locuteurs (l'âge, la trajectoire développementale et la langue) constitue un défi pour les modèles de la résolution d'anaphore. Dans cette étude, nous nous sommes intéressées aux stratégies d'interprétation des enfants francophones de 4 ans au développement langagier typique d'une part, et présentant un retard de langage d'autre part.

\subsection{Influence de l'âge sur les stratégies d'interprétation pronominale}


Différentes hypothèses peuvent être proposées quant au développement des stratégies d'interprétation pronominale chez les enfants. Suivant une première hypothèse, du fait de leurs capacités cognitives limitées (par exemple, une mémoire de travail plus réduite), les enfants utiliseraient moins d'indices linguistiques que les adultes pour interpréter une phrase (voir par exemple, Snedeker et Trueswell, 2004). Dans le cas de l'interprétation d'un pronom, les enfants commenceraient par appliquer une stratégie cognitive simple telle qu'une préférence pour le premier ou le dernier référent mentionné (Wykes, 1983). On peut aussi faire l'hypothèse que ce n'est pas le nombre d'indices pris en compte lors de l'interprétation d'un pronom qui distinguerait les enfants des adultes mais la pondération de ces indices. Un indice pertinent pour les adultes ne le serait pas nécessairement pour les enfants, et inversement (Arnold, BrownSchmidt \& Trueswell, 2007 ; Sekerina, 2015). Enfin, nous pouvons faire l'hypothèse que les enfants sont sensibles aux mêmes indices et de la même manière que les adultes, mais qu'ils sont plus lents pour prendre en compte ces indices lors de la résolution du pronom (Hartshorne, Nappa \& Snedeker, 2014).

Les récentes études expérimentales tendent à montrer que pour interpréter un pronom, les jeunes enfants, même s'ils sont plus lents que les adultes, n'appliquent pas une stratégie cognitive simple mais prennent en considération plusieurs indices linguistiques. Song et Fischer $(2005,2007)$ ont réalisé une série d'expériences avec la technique du regard préférentiel afin d'observer les stratégies d'interprétation des enfants anglophones de 2 ans et demi et de 3 ans pour interpréter un pronom anaphorique tel que he en (1b). Ils observent que dès 2 ans et demi, les enfants préfèrent comme antécédent du pronom le référent discursivement saillant (l'alligator en [1]) du fait qu'il est le premier référent mentionné, sujet et pronominalisé (donc mentionné plusieurs fois) dans le contexte discursif qui le précède (1a).

(1) a. On a sunny day, the alligator went outside. And he went to the tiger's yard.

b. And what did he find?

'Par une journée ensoleillée, l'alligator est sorti. Et il est allé dans le jardin du tigre.

Et qu'est-ce qu'il a trouvé?'

Dans une expérience d'enregistrement des mouvements oculaires pendant l'exploration d'une scène visuelle (paradigme dit du monde visuel), Hartshorne et al. (2014) observent que les enfants anglophones de 5 ans préfèrent comme antécédent d'un pronom anaphorique ambigu tel que she en (2), le premier référent mentionné et sujet (Emily en [2]). Cependant, cette préférence apparaît tardivement dans le traitement, les enfants sont plus lents que les adultes à prendre en compte cet indice linguistique pour interpréter le pronom.

(2) Emily went to school with Hannah. She read ten books.

'Emily est allée à l'école avec Hannah. Elle a lu dix livres.'

À nouveau avec le paradigme du monde visuel, Pyykkönen, Matthews et Järvikivi (2010) observent que les enfants anglophones de 3 ans préfèrent le sujet et premier référent mentionné (le panda en [3]) comme antécédent du pronom ambigu he en (3) et, comme dans l'expérience de Hartshorne et al. (2014), cet effet apparaît tardivement dans le traitement. D'autre part, ils observent que les enfants sont aussi sensibles à la transitivité du verbe lorsqu'ils interprètent le pronom. Les enfants regardent plus souvent le second référent et objet (le perroquet en [3]) avec des verbes hautement transitifs tels que "frapper" qu'avec des verbes moins transitifs tels que "voir". Ce dernier résultat montre que les enfants sont capables de prendre en compte plus d'un indice linguistique pour interpréter le pronom (ici le rôle grammatical et la transitivité du verbe).

(3) The panda hit the parrot near the hut. Do you know what happened next? He...

'Le panda a frappé le perroquet près de la cabane. Savez-vous ce qui s'est passé ensuite? Il...'

Enfin, toujours avec le paradigme du monde visuel, Järvikivi, Pyykkönen, Schimke, Colonna et Hemforth (2014) ont montré que les enfants germanophones de 4 ans préfèrent, eux aussi, un référent sujet comme antécédent d'un pronom ambigu et que cette préférence apparaît tardivement dans le traitement. De plus, ils observent que cette préférence pour le sujet augmente lorsque celui-ci est focalisé dans une construction clivée, suggérant à nouveau que les jeunes enfants sont sensibles à l'interaction de différents indices linguistiques lors de la résolution de pronom.

\subsection{Influence de la trajectoire développementale sur les stratégies d'interprétation pronominale}

Les études sur le traitement des formes pronominales des enfants au développement langagier atypique sont nettement moins nombreuses. De plus, la majorité de ces études se sont penchées sur les difficultés que les enfants au développement langagier atypique rencontrent lors de la production (voir par exemple, Tuller, Delage, Monjauze, Piller \& Barthez, 2011), et très peu se sont intéressées à leurs difficultés lors de la compréhension de pronoms. D'autre part, à notre connaissance aucune ne porte sur la compréhension des pronoms sujet. En effet, les études existantes se sont concentrées sur le traitement des pronoms objet. Van der Lely et Stollwerck (1997) ont réalisé une étude sur la compréhension des pronoms clitiques objet chez des enfants anglophones avec et sans trouble spécifique du langage. Ils observent que le groupe d'enfants avec trouble spécifique du langage (TSL), âgés de 9 à 12 ans, réussit moins bien la 
tâche de jugement de paires phrase-image (avec des phrases telles que [4]) que les enfants tout-venant plus jeunes (âgés de 5 à 9 ans).

(4) Is Mowgli tickling him?

'Est-ce que Mowgli le chatouille?'

Plus récemment, dans une étude utilisant l'enregistrement des mouvements oculaires avec le paradigme du monde visuel, Léger (2017) s'est intéressée à la compréhension du pronom clitique objet en français chez des enfants avec TSL âgés de 7 à 11 ans et des enfants tout-venant du même âge. Elle observe des difficultés d'interprétation du pronom clitique des enfants avec TSL comparés aux enfants tout-venants. Alors que ces derniers, après avoir entendu le pronom dans une phrase telle que (5), regardent majoritairement l'image cible (l'image d'un camion dans l'exemple [5]), les enfants avec TSL regardent indifféremment l'image cible et l'image d'un distracteur non apparié en genre avec la cible (l'image d'une voiture dans l'exemple [5]).

(5) Maman le conduit facilement, le camion.

Ces résultats soulèvent de nombreuses questions dont la nature exacte des difficultés que rencontrent les enfants au développement langagier atypique lors de l'interprétation des pronoms.

\subsection{Influence de la langue sur les stratégies d'interprétation pronominale}

L'interprétation des pronoms semble également être influencée par la langue des locuteurs. Hemforth et al. (2010) ont observé qu'alors que dans une phrase telle que (6), les locuteurs germanophones préfèrent le sujet de la proposition principale comme antécédent du pronom ambigu er, les locuteurs francophones préfèrent l'objet comme antécédent du pronom "il" en (7).

(6) Der Briefträger hat den Piraten getroffen, bevor er nach Hause ging.

(7) Le facteur a rencontré le pirate avant qu'il rentre à la maison.

Nous faisons l'hypothèse que cette différence est due à l'existence en français, et pas en allemand, d'une construction alternative non ambiguë. En effet, dans l'exemple (8), la proposition subordonnée "avant de rentrer à la maison" se rapporte obligatoirement au sujet de la proposition principale (le facteur en [8]). Suivant un principe gricéen, les locuteurs francophones inféreraient que lorsque c'est la construction ambiguë (7) qui est utilisée, c'est pour signifier que la subordonnée se rapporte à l'objet (pour une discussion voir Baumann, Konieczny \& Hemforth, 2014 ; Colonna, Schimke \& Hemforth, 2014).

(8) Le facteur a rencontré le pirate avant de rentrer à la maison.

En allemand, la préférence pour le sujet observée chez les adultes est présente chez les enfants dès 4 ans (voir section 1.1, Järvikivi \& al., 2014). Est-ce que comme les enfants allemands, les enfants francophones ont la même préférence d'interprétation du pronom que les adultes, à savoir une préférence pour l'objet (le pirate en [7]) ? Autrement dit, est-ce que la différence inter-langue observée entre les adultes francophones et germanophones est déjà présente chez les enfants de 4 ans?

\section{Notre étude}

Le but de notre étude est de tester la compréhension des constructions ambiguë et non ambiguë, réciproquement (9) et (10), par des enfants francophones de 4 ans présentant ou pas un retard de langage.

(9) Le lapin chatouille le renard avant qu'il imagine un jeu rigolo. (B)

(10) Le lapin chatouille le renard avant d'imaginer un jeu rigolo. (B)

Pour ce faire, nous avons utilisé le paradigme du monde visuel qui consiste à faire écouter au participant un énoncé oral faisant référence à certains éléments représentés dans une scène visuelle pendant que ses mouvements oculaires sont enregistrés. Dans cette expérience, nous présentions de courtes vidéos représentant les deux référents accomplissant l'action décrite dans la phrase orale test (B dans les exemples). Avec la vidéo représentée dans la figure 1, les enfants entendaient des phrases telles que (11). Le pattern d'exploration visuelle des enfants à partir du moment où ils entendent le pronom ambigu permet d'observer en temps réel quel référent est considéré comme antécédent.

(11) Voici le lapin et le renard. (A)

Le lapin chatouille le renard avant qu'il imagine un jeu rigolo. (B)

Mais ensuite le lapin se met à pleurer. (C) 
A

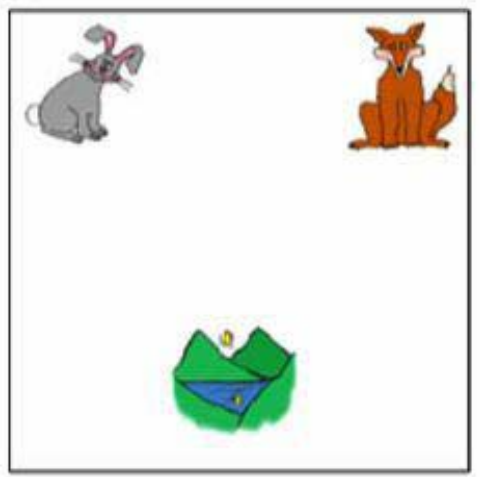

B

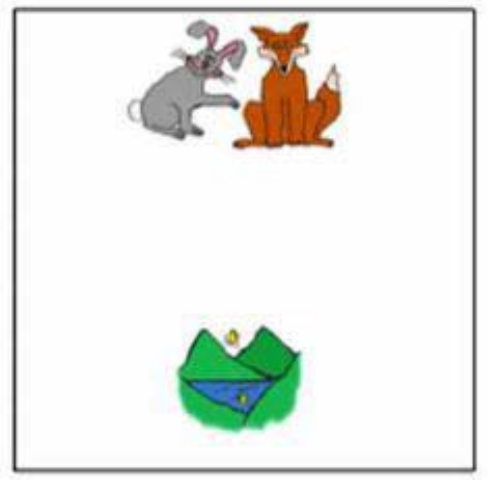

C

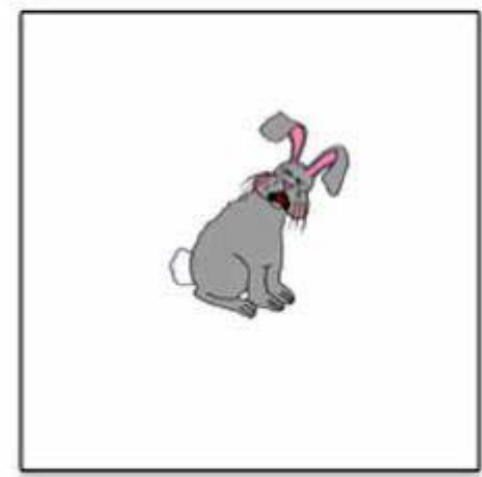

Figure 1 : Représentation schématique d'une vidéo expérimentale. Les images $\mathrm{A}, \mathrm{B}$ et $\mathrm{C}$ correspondent respectivement aux phrases $\mathrm{A}, \mathrm{B}$ et $\mathrm{C}$ dans les exemples.

Nous voulons observer d'une part, si les enfants francophones de 4 ans interprètent la construction infinitive (10) comme non ambiguë. Dans ce cas, ils devraient interpréter que c'est le lapin qui imagine un jeu rigolo et nous devrions observer plus de regards pour le lapin que pour le renard. D'autre part, nous souhaitons observer si dans la construction ambiguë (9), les enfants montrent déjà la préférence pour l'objet observée chez les adultes. Dans ce cas, les regards devraient être plus nombreux sur le renard que sur le lapin.

Nous avons par ailleurs testé la compréhension de ces constructions chez un groupe d'enfants présentant un retard de langage. À notre connaissance, la compréhension d'un pronom sujet ambigu par des enfants au développement langagier atypique n'a pas encore été étudiée. De même, l'interprétation d'une construction infinitive telle que (10), dont l'interprétation correcte fait appel aux compétences grammaticales, n'a jamais fait l'objet d'investigation avec des enfants au développement langagier atypique.

\subsection{Méthode}

\subsubsection{Participants}

\section{Enfants tout-venant}

Trente enfants monolingues francophones, âgés de 3 ans 10 mois à 4 ans 10 mois (moyenne : 4 ans 6 mois, écart-type = 0.30 ) ont participé à l'expérience. Les enfants étaient tous en moyenne section de maternelle. Ils ne présentaient ni pathologie ou retard de langage, ni pathologie neurologique ou cognitive, ni atteinte visuelle et/ou auditive noncorrigée. Nous avons par ailleurs demandé aux enseignant.e.s de nous signaler les enfants éventuellement en avance ou en retard par rapport à la classe de moyenne section afin de ne pas les inclure dans l'étude. Aucun enfant ne se trouvait dans cette situation. Les parents ont donné leur consentement avant la participation à l'étude de leur enfant.

\section{Enfants avec retard de langage}

Vingt-sept enfants monolingues francophones suivis en orthophonie pour un retard de langage oral avec suspicion de trouble spécifique du langage oral ont participé à l'expérience. Un enfant a été exclu des analyses car la perte de données oculaires était supérieure à $50 \%$ en raison de mouvements excessifs durant l'expérience. Les analyses rapportées ici portent donc sur 26 enfants avec retard de langage. Ils étaient âgés de 4 ans 0 mois à 6 ans 8 mois (moyenne : 5 ans 3 mois, écart-type $=0.80$ ) et scolarisés de la moyenne section de maternelle au CP. En dehors du retard de langage oral, ils ne présentaient ni pathologie neurologique ou cognitive, ni atteinte visuelle et/ou auditive non-corrigée. En revanche, comme c'est souvent le cas, nombre de ces enfants présentaient des troubles associés de la fluence et/ou un retard de parole avec des dysfonctionnements articulatoires. Ce qui n'a pas été pris en considération pour notre étude, la tâche utilisée ne sollicitant pas les capacités expressives mais uniquement les compétences réceptives.

\subsubsection{Matériel}

Les vidéos utilisées ont été adaptées de l'étude de Järvikivi et al. (2014). L'expérience comportait 20 vidéos expérimentales, 10 vidéos de remplissage et deux vidéos d'entraînement. Quatre versions de chaque vidéo expérimentale ont été créées comme représenté dans le tableau 1. L'ordre de présentation des animaux dans la phrase de présentation (A) était contrôlé, soit la phrase commençait par l'animal à gauche sur l'écran, soit par l'animal à droite. Le type de construction dans la phrase test (B) variait, soit la forme infinitive était testée, soit la construction avec un 
pronom ambigu. Le matériel a été distribué dans quatre listes expérimentales suivant un carré latin de façon à ce que chaque enfant voie toutes les conditions expérimentales mais jamais plusieurs fois le même item.

Tableau 1. Exemple d'un item expérimental dans les quatre conditions. A, B et $\mathrm{C}$ correspondent aux différentes images représentées Figure 1.

\begin{tabular}{|c|c|c|}
\hline A & B & C \\
\hline Voici le lapin et le renard. & $\begin{array}{r}\text { Le lapin chatouille le renard } \\
\text { avant qu'il imagine un jeu rigolo. }\end{array}$ & \multirow{2}{*}{$\begin{array}{c}\text { Mais ensuite le lapin se } \\
\text { met à pleurer. }\end{array}$} \\
\cline { 1 - 2 } Voici le lapin et le renard. & $\begin{array}{r}\text { Le lapin chatouille le renard } \\
\text { avant d'imaginer un jeu rigolo. }\end{array}$ & \\
\hline Voici le renard et le lapin. & $\begin{array}{r}\text { Le lapin chatouille le renard } \\
\text { avant qu'il imagine un jeu rigolo. }\end{array}$ & \\
\hline Voici le renard et le lapin. & $\begin{array}{r}\text { Le lapin chatouille le renard } \\
\text { avant d'imaginer un jeu rigolo. }\end{array}$ & \\
\hline
\end{tabular}

Les vidéos avaient toutes le patron suivant. Elles mettent en scène un couple d'animaux et un troisième élément distracteur, un lieu (un lac dans la figure 1). Elles commencent par la phrase de présentation (A) qui introduit les deux protagonistes. Puis, vient la phrase test (B) constituée de la proposition principale dans laquelle un des deux protagonistes réalise une action sur l'autre et de la proposition subordonnée qui contient ou non le pronom ambigu. La vidéo se termine par une phrase pour conclure la saynète. Quinze couples d'animaux ont été utilisés, chaque couple était du même genre (féminin ou masculin). Chaque couple était utilisé dans deux vidéos différentes dans lesquelles les rôles d'agent et de patient étaient inversés. De même, chaque verbe de la proposition principale était utilisé deux fois. Il s'agissait de verbes transitifs représentant une action concrète normalement connue des enfants (chatouille, gratte, embrasse, ...). Quant au verbe dans la proposition subordonnée, il commençait par une voyelle afin de désambiguïser le nombre du pronom ambigu (la liaison étant obligatoire dans le cas d'un pronom pluriel). Le matériel a été lu par une locutrice native francophone et enregistré en chambre sourde. La technique du cross-splicing a été utilisée entre la proposition principale et la subordonnée pour contrebalancer d'éventuels effets prosodiques. Le matériel audio a été superposé aux images pour constituer les vidéos de la façon suivante. La première image $\mathrm{A}$ où les animaux se trouvent dans les coins gauche et droit (en haut ou en bas) de l'écran et le distracteur au milieu (en bas ou en haut) apparaît une seconde puis la phrase de présentation est jouée. Huit cent millisecondes après commence la proposition principale en même temps que la deuxième image $\mathrm{B}$ s'affiche à l'écran suivie d'un silence de $200 \mathrm{~ms}$ avant la proposition subordonnée. Au moment du silence avant la subordonnée, les animaux reviennent rapidement à leur position initiale (à nouveau, image A). Un dernier silence de $1600 \mathrm{~ms}$ a été introduit avant la phrase de conclusion. La phrase de conclusion coïncide avec la dernière image $\mathrm{C}$ représentant un des deux animaux, les deux animaux ensemble ou encore le distracteur.

\subsubsection{Procédure}

Tous les enfants ont été testés individuellement. Les enfants tout-venant ont été testés dans leur école, les enfants avec retard de langage en cabinet d'orthophonie. Pendant la passation, l'enfant était installé à une distance oeil-écran de 60 $\mathrm{cm}$ et l'expérimentatrice assise à côté. On commençait par montrer un diaporama présentant aux enfants les animaux de l'expérience. Une fois les animaux présentés, on leur expliquait qu'ils/elles allaient voir de petites vidéos avec ces animaux. Chaque enfant voyait une des quatre listes constituée de 30 vidéos. Chaque couple d'animaux apparaissant deux fois au cours de l'expérience, l'ordre de présentation a été décidé de façon à ce qu'un même couple n'apparaisse jamais dans deux items à la suite mais toujours espacé d'au moins cinq items. D'autre part, un item de remplissage était inséré tous les deux items expérimentaux. Les mouvements oculaires des enfants étaient enregistrés grâce à l'oculomètre Tobii T120 avec un enregistrement binoculaire et une fréquence d'échantillonnage de 120 Hertz. Après la passation, tous les enfants recevaient un diplôme de petit.e chercheur.se, les enfants testés en cabinet d'orthophonie recevaient aussi un livret de jeux.

\subsection{Résultats}




\subsubsection{Analyse des données}

Les analyses de données ont été réalisées avec les packages eyetrackingR (Dink \& Ferguson, 2018) et lme4 (Bates, Maechler, Bolker \& Walker, 2015) de R (R Core Team, 2014). Nous avons défini des aires d'intérêt correspondant à la position de chaque animal sur l'écran afin de déterminer, pour chaque segment de temps de $50 \mathrm{~ms}$, si les enfants regardent l'animal correspondant au sujet $(\mathrm{N} 1)$ ou à l'objet $(\mathrm{N} 2)$ de la proposition principale. La variable dépendante considérée est la proportion de regards sur N1 par rapport à N2 (soit la somme des fixations oculaires sur N1 divisée par la somme des fixations sur N1 et N2). La fenêtre de temps pour laquelle nous avons examiné la proportion de regards sur N1 commence avec le début du pronom (pour la condition ambiguë) ou avec le début du "de" dans la conjonction "avant de" (pour la condition non ambiguë) et se termine 3 secondes après (voir figures 2 et 3 ). Les essais pour lesquels la perte de données était supérieure à $25 \%$ étaient exclus des analyses. Après transformation logarithmique de la variable dépendante, les données ont été analysées avec des modèles linéaires à effets mixtes. Ce type de modèles statistiques présente l'avantage de pouvoir inclure simultanément les deux effets aléatoires croisés des participants et des items (Baayen, Davidson \& Bates, 2008 ; Barr, Levy, Scheepers \& Tily, 2013). Nous avons inclus dans notre modèle le type de construction (ambiguë versus non ambiguë) comme effet fixe, les participants et les items dans les intercepts aléatoires. Dans les pentes aléatoires, nous avons inclus la structure des effets aléatoires maximale justifiée par les données (Jaeger, 2011). Les khi-carrés du rapport de vraisemblance et les valeurs-p pour l'effet fixe ont été obtenus grâce à la fonction drop 1 du package lme4. Nous avons ainsi réalisé des analyses pour chaque fenêtre de temps de $400 \mathrm{~ms}$ comprise entre 0 et $2800 \mathrm{~ms}$ après le début du pronom (ou de "de" suivant la condition).

\subsubsection{Enfants tout-venant}

La figure 2 présente la proportion de regards sur N1 des enfants tout-venant à partir du début du pronom pour la condition ambiguë (construction finie) et à partir du début de "de" pour la condition non ambiguë (construction non finie). Les analyses révèlent que la proportion de regards sur N1 est statistiquement différente dans les deux conditions dans la fenêtre de temps comprise entre 1200 et $1600 \mathrm{~ms}$ (Estimate $=-0.861, \mathrm{SE}=0.04, \mathrm{t}=-2.015, \mathrm{X} 2(1)=3.96 ; \mathrm{p}<0.5$ ). Comme attendu, la proportion de regards sur N1 est plus élevée dans la condition avec la construction infinitive qu'avec la construction avec le pronom ambigu pour laquelle les regards pour $\mathrm{N} 1$ par rapport à $\mathrm{N} 2$ se situent autour de $50 \%$.

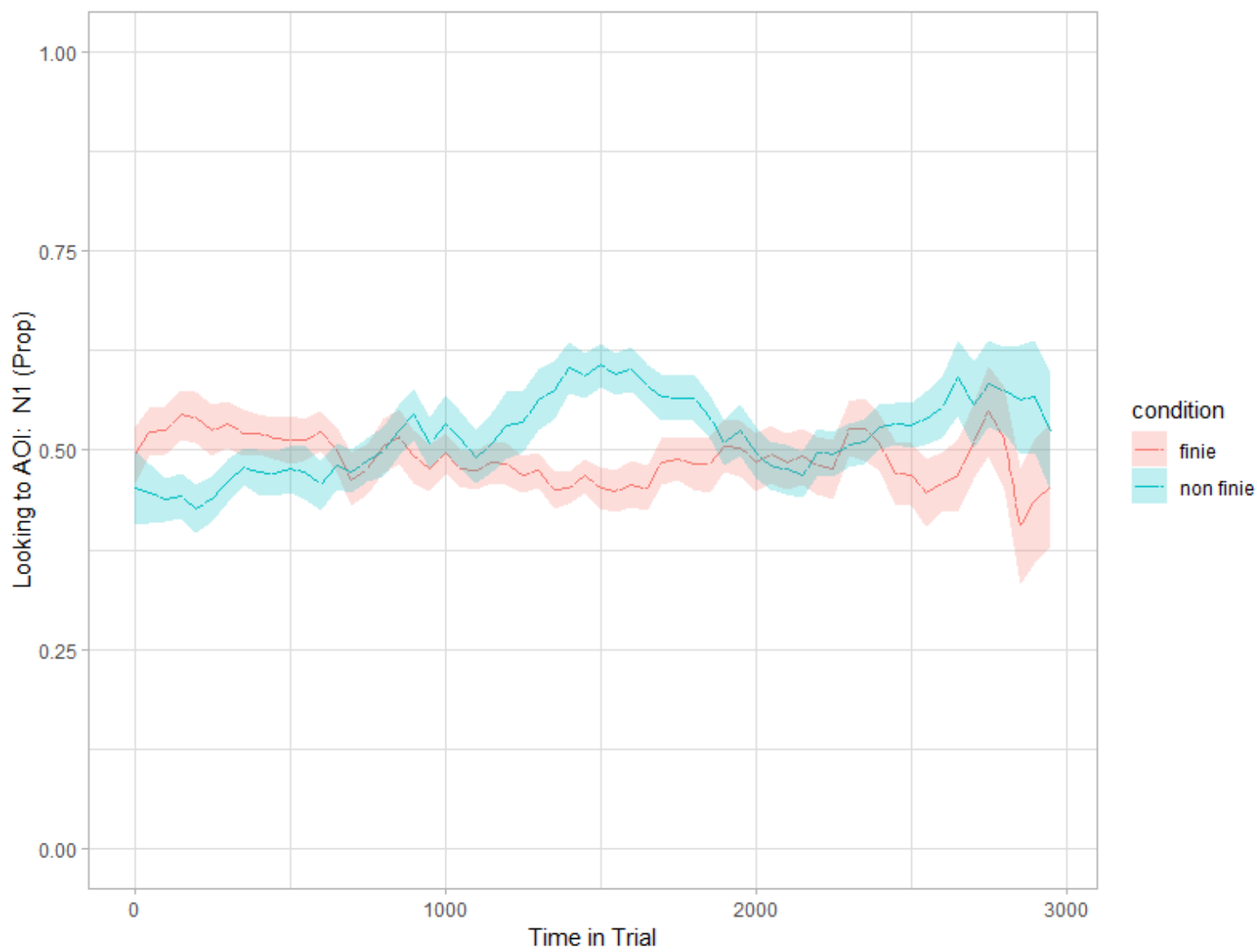

Figure 2. Proportion de fixations oculaires sur N1 des enfants tout-venant en fonction du temps et du type de construction. 


\subsubsection{Enfants avec retard}

La figure 3 présente la proportion de regards sur N1 des enfants avec retard de langage à partir du début du pronom pour la condition ambiguë (construction finie) et à partir du début de "de" pour la condition non ambiguë (construction non finie). Pour chaque fenêtre de temps de $400 \mathrm{~ms}$ examinée, les analyses n'ont pas révélé d'effet statistiquement significatif du type de construction sur la proportion de fixations sur N1. Dans les deux conditions, la proportion de regards sur N1 par rapport à N2 est autour de 50\%. Les enfants avec retard de langage semblent regarder aléatoirement l'un ou l'autre des référents quelle que soit la construction.

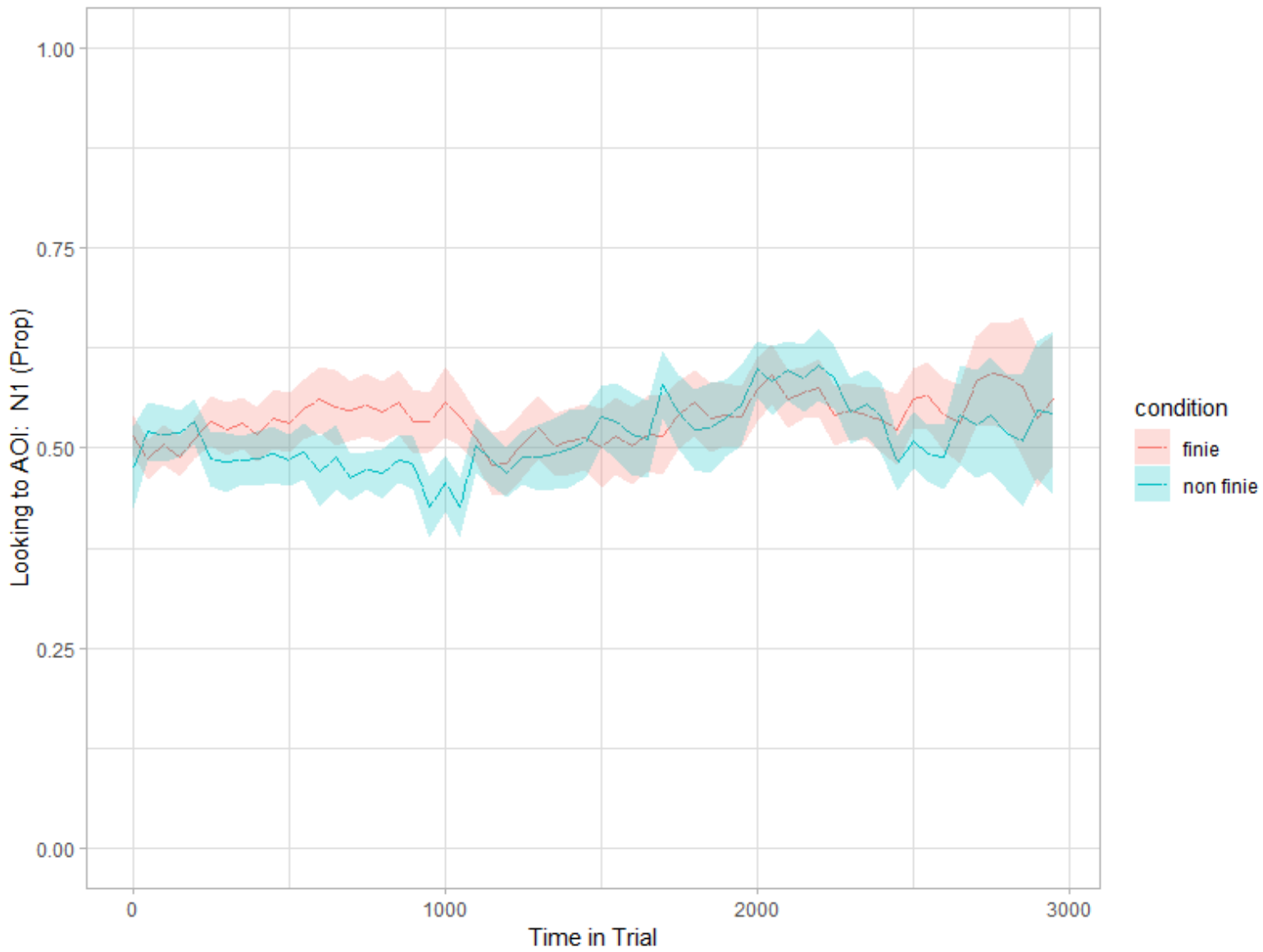

Figure 3. Proportion de fixations oculaires sur N1 des enfants avec retard de langage en fonction du temps et du type de construction.

\section{Discussion}

Les résultats suggèrent que les enfants tout-venant de 4 ans commencent à distinguer les constructions testées (reproduites en [12] et [13]). Nous observons en effet que les regards sur le sujet de la proposition principale (le lapin dans les exemples) sont plus nombreux dans les constructions infinitives telles que (13) que dans les constructions avec un pronom anaphorique telles que (12).

(12) Le lapin chatouille le renard avant qu'il imagine un jeu rigolo.

(13)Le lapin chatouille le renard avant d'imaginer un jeu rigolo.

Dans le cadre d'une autre étude, un questionnaire avec des adultes a été réalisé pour tester leur interprétation de phrases équivalentes à (13) telles que (14) (Wang, Vincent, Schimke \& Colonna, 2019). Les résultats révèlent que les adultes francophones interprètent dans plus de $99 \%$ des cas, la subordonnée infinitive comme coréférentielle au sujet de la principale (i.e. que c'est Eric qui range ses affaires dans l'exemple [14]). Les enfants tout-venant de notre étude se distinguent donc des adultes puisque leur proportion de regard pour N1 avec les constructions non finies telle qu'en (13) est encore loin des 100\% (voir Figure 2). Si ce type de structure est non ambiguë pour les adultes, ce n'est vraisemblablement pas encore le cas pour les enfants de 4 ans.

(14) Eric a observé Albert avant de ranger ses affaires.

En anglais aussi, il existe une construction non finie qui permet d'exprimer sans ambiguïté la coréférence de la subordonnée temporelle avec le sujet de la proposition principale. Les études menées sur ce type de construction corroborent nos résultats. En effet, dans des phrases telles que (15), alors que les adultes interprètent sans ambiguïté la 
coréférence de la temporelle au sujet de la principale, ce n'est pas encore le cas des enfants anglophones de 4 ans (Gerard, Lidz, Zuckerman et Pinto, 2017, 2018).

(15) Dora washed Diego before eating the red apple.

\section{'Dora a arrosé Diego avant de manger la pomme rouge.'}

Les enfants francophones se distinguent aussi des adultes dans leur interprétation de la construction avec un pronom ambigu en (12). En effet, comme vu en introduction, dans des phrases telles que (16), les adultes francophones interprètent préférentiellement le pronom ambigu comme se référant à l'objet de la proposition principale (Hemforth \& al., 2010 ; Schimke, de la Fuente, Hemforth \& Colonna, 2018). Nos résultats révèlent que ce n'est pas encore le cas des enfants de 4 ans qui ne montrent pas de préférence pour l'un ou l'autre des référents dans ce type de construction. Nous savons que chez les adultes, ce n'est pas seulement l'existence d'une construction alternative non ambiguë dans la langue qui influence l'interprétation du pronom mais également la fréquence d'usage de la construction alternative. En anglais, nous avons vu qu'il existait aussi une forme alternative non ambiguë (voir exemple [15]), cette construction est néanmoins moins fréquente qu'en français (voir Schulz, Burnett \& Hemforth, 2019 pour une analyse de corpus). Dans des phrases telles que (17) (dont l'alternative non ambiguë est donnée en [18]), les adultes anglophones préfèrent le sujet de la principale comme antécédent du pronom ambigu. Cette préférence est néanmoins moins marquée qu'en allemand où il n'existe pas du tout de construction alternative non ambiguë (pour une discussion, voir Baumann \& al., 2014 ; Hemforth \& al., 2010). La fréquence d'exposition à une construction alternative non ambiguë semble donc jouer un rôle dans les stratégies d'interprétation du pronom ambigu.

(16) Le facteur a rencontré le pirate avant qu'il rentre à la maison.

(17) The postman met the pirate before he went home.

(18) The postman met the pirate before going home.

Concernant les enfants, il est possible qu'à 4 ans, ils n'aient pas encore suffisamment été exposés à la construction non ambiguë pour qu'elle influence leur résolution du pronom. Il faut cependant noter que s'ils ne montrent pas la préférence pour l'objet observée chez les adultes francophones, ils n'exhibent pas non plus la préférence pour le sujet observée chez les enfants germanophones (Järvikivi \& al., 2014) et anglophones (Hartshorne \& al., 2014 ; Pyykkönen $\&$ al., 2010 ; Song \& Fischer, 2005, 2007). On peut donc faire l'hypothèse que la construction alternative non ambiguë commence à influencer leur résolution du pronom mais que ce n'est pas le seul facteur en jeu. Nous avons vu en effet en introduction que les enfants dès 4 ans prenaient en compte plusieurs indices linguistiques pour interpréter un pronom. En particulier, comme les adultes, ils sont sensibles à la saillance discursive des référents. Dans l'exemple (12) le lapin est le référent discursivement saillant du fait qu'il est premier mentionné, sujet et agent. L'absence de préférence pour l'un ou l'autre des référents observée en (12) peut donc être le résultat de deux facteurs, la saillance discursive qui favorise le premier référent et sujet d'une part, l'influence de la construction alternative qui favorise le second référent et objet d'autre part. Même si chez les adultes aussi ces deux facteurs sont vraisemblablement à l'oeuvre, le poids de la construction alternative dans l'interprétation est plus important que chez les enfants de 4 ans, ces derniers ayant été moins exposés à la langue (et à ce type de construction). Afin de mieux comprendre le rôle et le poids de la fréquence d'exposition, nous prévoyons de tester l'interprétation des pronoms ambigus dans des phrases telles que (12) chez des enfants d'âges différents, des enfants de 6 ans, 8 ans et 10 ans. La proportion d'interprétation en faveur de l'objet devrait augmenter avec l'âge (et la fréquence d'exposition) jusqu'à observer la même préférence que chez les adultes.

Nos résultats montrent que les enfants tout-venant de 4 ans se distinguent des adultes mais également des enfants présentant un retard de langage (à noter que les enfants de ce groupe étaient en moyenne un peu plus âgés que les enfants dans le groupe tout-venant). Alors que les enfants tout-venant commencent à montrer une préférence pour le sujet dans la construction infinitive non ambiguë, les enfants avec un retard de langage ne regardent pas préférentiellement l'un ou l'autre des référents et ce, dans les deux types de constructions. Là aussi, des données complémentaires doivent être recueillies afin de déterminer s'il y a un âge à partir duquel les enfants avec un retard de langage (ou plus âgés, avec un trouble spécifique du langage diagnostiqué) se comportent comme les enfants toutvenant. Si l'écart entre les enfants tout-venant et les enfants avec un développement langagier atypique se confirme, l'absence d'interprétation de la coréférence d'une subordonnée infinitive telle qu'en (13) au sujet de la principale pourrait être un indice de plus à prendre en compte dans le diagnostic d'un trouble du développement langagier.

\section{Références bibliographiques}

Arnold, J. E., Brown-Schmidt, S., \& Trueswell, J. (2007). Children's use of gender and order-of-mention during pronoun comprehension. Language and cognitive processes, 22(4), 527-565.

Baayen, H., Davidson, D., \&. Bates, D. (2008). Mixed-effects modeling with crossed random effects for subjects and items. Journal of Memory and Language, 59(4). 390-412. 
Barr, D., Levy, R., Scheepers, C., \& Tily, H. (2013). Random effects structure for confirmatory hypothesis testing: Keep it maximal. Journal of Memory and Language, 68(3). 255-278.

Bates, D., Maechler, M., Bolker, B., \& Walker, S. (2015). Fitting Linear Mixed-Effects Models Using lme4. Journal of Statistical Software, 67(1), 1-48.

Baumann, P., Konieczny, L., \& Hemforth, B. (2014). Conversational implicatures in anaphora resolution: Alternative constructions and referring expressions. In B. Hemforth, B., Schmiedtovà, \& C. Fabricius-Hansen (Eds.), Psycholinguistic approaches to meaning and understanding across languages (pp. 197-212). Studies in theoretical psycholinguistics. Munich: Springer.

Colonna, S., Schimke, S., \& Hemforth, B. (2014). Information structure and pronoun resolution in German and French: Evidence from the visual-world paradigm. In B. Hemforth, B., Schmiedtovà, \& C. Fabricius-Hansen (Eds.), Psycholinguistic approaches to meaning and understanding across languages (pp. 175-195). Studies in theoretical psycholinguistics. Munich: Springer.

de la Fuente, I., Hemforth, B., Colonna, S., \& Schimke, S. (2016). The role of syntax, semantics, and pragmatics in pronoun resolution: A cross-linguistic overview. In A. Holler \& K. Suckow (Eds.), Experimental Perspectives on Anaphora Resolution, Linguistische Arbeiten 563 (pp. 11-31). Berlin/Boston: De Gruyter Mouton.

Dink J., \& Ferguson, B. (2018). eyetrackingR. R package version 0.1.8, http://www.eyetracking-R.com.

Gerard, J., Lidz, J., Zuckerman, S., \& Pinto, M. (2017). Similarity-Based Interference and the Acquisition of Adjunct Control. Frontiers in Psychology, 8, 1822. doi: 10.3389/fpsyg.2017.01822

Gerard, J., Lidz, J., Zuckerman, S., \& Pinto, M. (2018). The acquisition of adjunct control is colored by the task. Glossa: A Journal of General Linguistics, 3(1), 75. doi: 10.5334/gjgl.547

Hartshorne, J., Nappa, R., \& Snedeker, J. (2014). Development of the First-Mention Bias. Journal of Child Language, 15, 1-24.

Hemforth, B., Konieczny, L., Scheepers, C., Colonna, S., Schimke, S., Baumann, P., \& Pynte, J. (2010). Language specific preferences in anaphor resolution: Exposure or gricean maxims? In S. Ohlsson \& R. Catarambone (Eds.), Proceedings of the 32nd annual conference of the cognitive science society (pp. 2218-2223). Austin, TX: Cognitive Science Society.

Jaeger, F. (2011). More on random slopes and what it means if your effect is not longer significant after the inclusion of random slopes. Post to HLP/Jaeger lab blog. Consulté sur http://hlplab.wordpress.com/2011/06/25/more-on-random-slopes.

Järvikivi, J., Pyykkönen, P., Schimke, S., Colonna, S., \& Hemforth, B. (2014). Information structure cues for 4 year olds and adults: Tracking eye-movements to visually presented anaphoric referents. Language, Cognition and Neuroscience, 29(7), 877-892.

Léger, E. (2017). Interprétation des pronoms clitiques objets chez les enfants avec TSA et chez les enfants avec TSL. Etude comparative en suivi du regard. Thèse de doctorat, Université de Tours, France.

Pyykkönen, P., Matthews, D., \& Järvikivi, J. (2010). Three-year-olds are sensitive to semantic prominence during online language comprehension: A visual world study of pronoun resolution. Language and Cognitive Processes, 25, 115-129. doi:10.1080/01690960902944014

R Core Team (2014). R: A language and environment for statistical computing. R Foundation for Statistical Computing, Vienna, Austria. URL http://www.R-project.org/.

Sekerina, I. A. (2015). Online evidence for children's interpretation of personal pronouns. In L. Serratrice \& S. E. Allen (Eds.), The acquisition of reference (pp. 213-239). Amsterdam: John Benjamins.

Schimke, S., de la Fuente, I., Hemforth, B., \& Colonna S. (2018). First language influence on second language offline and online ambiguous pronoun resolution. Language Learning, 68(3), 744-779. doi:10.1111/lang.12293

Schulz, M., Burnett, H., \& Hemforth, B. (2019). A Rational Speech Act model of cross-linguistic differences in pronoun resolution preferences. Poster presented at CUNY Conference on Human Sentence Processing, Colorado Boulder.

Snedeker, J., \& Trueswell J. (2004). The developing constraints on parsing decisions: The role of lexical-biases and referential scenes in child and adult sentence processing. Cognitive Psychology, 49(3), 238-299.

Song, H.., \& Fisher, C. (2005). Who's “'she'”? Discourse prominence influences preschoolers' comprehension of pronouns. Journal of Memory \& Language, 52(1), 29-57. doi:10.1016/j.jml.2004.06.012

Song, H., \& Fisher, C. (2007). Discourse prominence effects on 2.5-year-old children's interpretation of pronouns. Lingua, 117, 1959-1987. doi:10.1016/j.lingua.2006.11.011

Tuller, L., Delage, H., Monjauze, C., Piller, A.-G., \& Barthez, M.-A. (2011). Clitic pronoun production as a measure of atypical language development in French. Lingua, 121. doiDOI: 10.1016/j.lingua.2010.10.008

van der Lely, H. K. J., \& Stollwerck, L. (1997). Binding theory and grammatical specific language impairment in children. Cognition, 62(3), 245-290. 
Wang, C.-C., Vincent, C., Schimke, S., \& Colonna, S. (2019). Interpretation strategies of anaphoric pronoun by second language learners. Manuscrit en préparation.

Wykes, T. (1983). The role of inferences in children's comprehension of pronouns. Journal of Experimental Child Psychology, 35 , 180-193. 